Pacific

Journal of

Mathematics

A COUNTEREXAMPLE TO THE ENERGY IDENTITY FOR SEQUENCES OF $\alpha$-HARMONIC MAPS

YUXIANG LI AND YoUdE WANG 


\title{
A COUNTEREXAMPLE TO THE ENERGY IDENTITY FOR SEQUENCES OF $\alpha$-HARMONIC MAPS
}

\author{
YUXIANG LI AND YOUDE WANG
}

We construct a closed Riemannian manifold $(N, h)$ and a sequence of $\alpha$ harmonic maps from $S^{2}$ into $N$ with uniformly bounded energy such that the energy identity for this sequence is not true.

\section{Introduction}

Let $(\Sigma, g)$ be a Riemann surface and $(N, h)$ be an $n$-dimensional smooth compact Riemannian manifold which is embedded in $\mathbb{R}^{K}$. Usually, we denote the space of Sobolev maps from $\Sigma$ into $N$ by $W^{k, p}(\Sigma, N)$, which is defined by

$$
W^{k, p}(\Sigma, N)=\left\{u \in W^{k, p}\left(\Sigma, \mathbb{R}^{K}\right): u(x) \in N \text { for a.e. } x \in \Sigma\right\} .
$$

For $u \in W^{1,2}(\Sigma, N)$, we define locally the energy density $e(u)$ of $u$ at $x \in \Sigma$ by

$$
e(u)(x)=\left|\nabla_{g} u\right|^{2}=g^{i j}(x) h_{\alpha \beta}(u(x)) \frac{\partial u^{\alpha}}{\partial x^{i}} \frac{\partial u^{\beta}}{\partial x^{j}} .
$$

The energy of $u$ on $\Sigma$, denoted by $E(u)$ or $E(u, \Sigma)$, is defined by

$$
E(u)=\frac{1}{2} \int_{\Sigma} e(u) d V_{g},
$$

and the critical points of $E$ are called harmonic maps. We know that a harmonic map $u$ satisfies

$$
\tau(u)=\Delta u+A(u)(\nabla u, \nabla u)=0,
$$

where $A$ is the second fundamental form of $N$ in $\mathbb{R}^{K}$. Harmonic maps are related very closely to minimal surface. It is well known that a harmonic map from $S^{2}$ into $N$ must be a branched conformal immersion in $N$.

Unfortunately, $E$ does not satisfy the Palais-Smale condition. From the viewpoint of calculus of variation, it is difficult to show the existence of harmonic maps from a 
surface. In order to obtain harmonic maps, Sacks and Uhlenbeck [1981] introduced the so-called $\alpha$-energy $E_{\alpha}$, instead of $L^{2}$ energy $E$, as

$$
E_{\alpha}(u)=\frac{1}{2} \int_{\Sigma}\left\{\left(1+|\nabla u|^{2}\right)^{\alpha}-1\right\} d V_{g},
$$

where we always assume that $\alpha>1$. It is well known that this $\alpha$-energy functional $E_{\alpha}$ satisfies the Palais-Smale condition. The critical points of $E_{\alpha}$ in $W^{1,2 \alpha}(\Sigma, N)$, called $\alpha$-harmonic maps, satisfy

$$
\Delta_{g} u_{\alpha}+(\alpha-1) \frac{\nabla_{g}\left|\nabla_{g} u_{\alpha}\right|^{2} \nabla_{g} u_{\alpha}}{1+\left|\nabla_{g} u_{\alpha}\right|^{2}}+A\left(u_{\alpha}\right)\left(d u_{\alpha}, d u_{\alpha}\right)=0 .
$$

The strategy of Sacks and Uhlenbeck is to employ a sequence of $\alpha$-harmonic maps to approximate a harmonic map as $\alpha$ tends to 1 . Hence, to show the existence of harmonic maps we need to study the convergence behavior of a sequence of $\alpha$-harmonic maps $u_{\alpha}$ with $E_{\alpha}\left(u_{\alpha}\right)<C$ from a compact surface $(\Sigma, g)$ into a compact Riemannian manifold $(N, h)$ without boundary. Generally, such a sequence converges weakly to a harmonic map in $W^{1,2}(\Sigma, N)$ and strongly in $C^{\infty}$ away from a finite set of points in $\Sigma$.

Concretely, let $\left\{u_{\alpha_{k}}\right\}$ be a sequence of $\alpha$-harmonic maps from $\Sigma$ into $N$ with uniformly bounded $\alpha$-energy, that is, $E_{\alpha_{k}}\left(u_{\alpha_{k}}\right)<\Lambda<\infty$. We assume that the sequence does not converge smoothly on $\Sigma$. By the theory of Sacks and Uhlenbeck, there exists a subsequence of $\left\{u_{\alpha_{k}}\right\}$, still denoted by $\left\{u_{\alpha_{k}}\right\}$, and a finite set $\mathscr{Y} \subset \Sigma$ such that the subsequence converges to a harmonic map $u_{0}$ in $C_{\mathrm{loc}}^{\infty}(\Sigma \backslash \mathscr{Y})$. We know that, at each point $p_{i} \in \mathscr{Y}$, the energy of the subsequence concentrates and the blowup phenomena occurs. Moreover, there exist point sequences $\left\{x_{i_{k}}^{l}\right\}$ in $\Sigma$ with $\lim _{k \rightarrow+\infty} x_{i_{k}}^{l}=p_{i}$ and scaling constant number sequences $\left\{\lambda_{i_{k}}^{l}\right\}$ with $\lim _{k \rightarrow+\infty} \lambda_{i_{k}}^{l} \rightarrow 0, l=1, \ldots, n_{0}$, such that

$$
u_{\alpha_{k}}\left(x_{i_{k}}^{l}+\lambda_{i_{k}}^{l} x\right) \rightarrow v^{l} \quad \text { in } C_{\mathrm{loc}}^{j}\left(\mathbb{R}^{2} \backslash \mathscr{A}^{i}\right),
$$

where all $v^{i}$ are nontrivial harmonic maps from $S^{2}$ into $N$, and $\mathscr{A}^{i} \subset \mathbb{R}^{2}$ is a finite set.

In order to explore and describe the asymptotic behavior of $\left\{u_{\alpha_{k}}\right\}$ at each blowup point, the following two problems arise naturally. The first is whether or not the energy identity holds true:

$$
\lim _{\alpha_{k} \rightarrow 1} E_{\alpha_{k}}\left(u_{\alpha_{k}}, B_{r_{0}}^{\Sigma}\left(p_{i}\right)\right)=E\left(u_{0}, B_{r_{0}}^{\Sigma}\left(p_{i}\right)\right)+\sum_{l=1}^{n_{0}} E\left(v^{l}\right) .
$$

Here, $B_{r_{0}}^{\Sigma}\left(p_{i}\right)$ is a geodesic ball in $\Sigma$ which contains only one blowup point $p_{i}$. The other is whether or not the necks connecting bubbles are some geodesics of finite length? 
Considerable progress has been made regarding these problems; let us now recall some main results on them. Chen and Tian [1999] considered a special sequence $\left\{u_{\alpha_{k}}\right\}$ with uniformly bounded $\alpha$-energy, for which every $u_{\alpha_{k}}$ is a minimizing $\alpha_{k}$-harmonic map and all maps $u_{\alpha_{k}}$ belong to a fixed homotopy class. They studied the convergence behavior of such a special sequence and provided a proof on the above energy identity. Later, for the same sequence, Li and Wang [2010a] gave another constructing proof on the energy identity, which is completely different from that given in [Chen and Tian 1999].

The energy identity for a minimax sequence of $\alpha$-harmonic maps has also been considered. Suppose that $A$ is a parameter manifold. Let $h_{0}: \Sigma \times A \rightarrow N$ be a continuous map, and $H$ be such a set of continuous maps $h: \Sigma \times A \rightarrow N$ that every $h \in H$ is homotopic to $h_{0}$ and satisfies $h(t) \in W^{1,2 \alpha}(\Sigma, N)$ for any fixed $t \in A$. Set

$$
\beta_{\alpha}(H)=\inf _{h \in H} \sup _{t \in A} E_{\alpha}(h(\cdot, t)) .
$$

It is known that there is at least a sequence $\left\{u_{\alpha_{k}}\right\}$, each $u_{\alpha_{k}}$ of which attains $\beta_{\alpha_{k}}(H)$, satisfies the energy identity as $\alpha_{k} \rightarrow 1$. For more details, we refer to [Jost 1991; Lamm 2010].

On the other hand, it should be pointed out that some effective methods have been established to successfully prove the energy identity and give a detailed description of the connecting necks for the heat flow of harmonic maps from a Riemann surface, or more generally, a sequence of maps from a Riemann surface with tension fields $\tau$ bounded in the sense of $L^{2}$ [Ding 1998; Ding and Tian 1995; Qing 1995; Qing and Tian 1997].

Recently, Li and Wang [2010b] studied the above problems on the sequences of $\alpha$-harmonic maps and obtained some results which can be summarized as follows. If the energy concentration phenomena appears for $\left\{u_{\alpha_{k}}\right\}$, one can prove a weak energy identity and a direct convergence relation between the blowup radius and the parameter $\alpha$, which ensures the energy identity and no-neck property. Li and Wang also showed that the necks converge to some geodesics and gave a length formula for the neck in the case where only one bubble appears.

Motivated by an example given by Duzaar and Kuwert [1998], Li and Wang [2010b] also constructed an $\alpha$-harmonic map sequence with uniformly bounded energy, for which the blowup phenomenon occurs and there exists at least a neck (geodesic) of infinite length. This answers negatively the second problem on $\alpha$ harmonic map sequence.

Although some mathematicians think that the energy identity for the sequence of $\alpha$-harmonic maps should also be true, up to now it has been unclear in general whether the energy identity for an $\alpha$-harmonic map sequence with bounded energy 
holds true or not. In this short paper, we will modify the construction in [ $\mathrm{Li}$ and Wang 2010b] to show that the energy identity is also not true.

On the other hand, a natural problem is whether the set of the values of energy for harmonic spheres in any given Riemannian manifold $(N, h)$ is discrete or not, since the bubbles produced in the convergence of a sequence of $\alpha$-harmonic maps from $(\Sigma, g)$ are always harmonic spheres.

We denote this set by

$$
\mathscr{E}(N, h)=\left\{E(u): u \text { is a harmonic map from } S^{2} \text { into }(N, h)\right\} .
$$

It is well known that if $(N, h)$ is the standard sphere $S^{2}$, we have

$$
\mathscr{E}(N, h)=\{4 k \pi: k=0,1, \ldots, n, \ldots\} .
$$

We also know from [Valli 1988] that if $(N, h)$ is the unitary group $U(n)$ with the standard metric, then the energy of harmonic maps $S^{2} \rightarrow U(n)$ can take as values only integral multiples of $8 \pi$. Some other energy gap phenomena on unitons were discussed in [Anand 1995; Dong 2002; Uhlenbeck 1989]. Some mathematicians conjectured that $\mathscr{E}(N, h)$ is a discrete set. Here, we will also give a counterexample to show that $\mathscr{E}(N, h)$ is not discrete.

\section{2. $\alpha$-harmonic maps}

Later, we will discuss the convergence behavior of some $\alpha$-harmonic map sequences with uniformly bounded $\alpha$-energy or $L^{2}$ energy. In fact, by discussing the convergence of $\alpha$-harmonic map sequences, Sacks and Uhlenbeck developed an existence theory on minimal surfaces in [Sacks and Uhlenbeck 1981; 1982]. In particular, they established the well-known $\epsilon$-regularity theorem on $\alpha$-harmonic maps and removal singularity theorem on harmonic maps [1981], which will be used repeatedly in the present paper.

Theorem 2.1. Let $D=D_{1}(0)=\{z:|z|<1\} \subset \mathbb{C}$ be a disk with radius 1 and $N$ be a Riemannian manifold. Assume that $u: D \rightarrow N$ satisfies Equation (1-1). Then there exists $\epsilon_{0}>0$ and $\alpha_{0}>1$ such that if $E(u, D)<\epsilon_{0}$ and $1 \leq \alpha \leq \alpha_{0}$, then we have

$$
\left\|\nabla^{k} u\right\|_{L^{\infty}\left(D_{1 / 2}\right)} \leq C(k) E(u, D) .
$$

Theorem 2.2. Assume that $u: D \backslash\{0\} \rightarrow N$ is a harmonic map with $E(u)<+\infty$. Then $u$ is a harmonic map from $D$ into $N$.

The above theorem tells us that, if $u$ is a harmonic map from $\mathbb{C} \backslash\left\{p_{i} \in \mathbb{C}: i=\right.$ $1,2, \ldots, l<\infty\}$ into $N$ with $E(u)<+\infty$, then $u$ can be viewed as a harmonic map from $S^{2}$ into $N$. 
Now, we can state more precisely the energy concentration of $\left\{u_{\alpha_{k}}\right\}$. Let $B_{t}^{\Sigma}(x)$ denote the geodesic ball of $\Sigma$ which is centered at $x$ and has geodesic radius $t$. By Theorem 2.1, the finite singular set of $\left\{u_{\alpha_{k}}\right\}$ can be defined precisely by

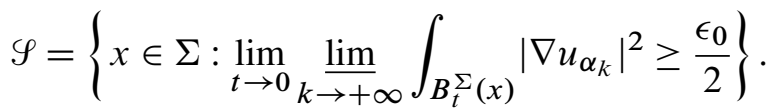

For any $\tilde{x}_{0} \notin \mathscr{Y}$, there exists $\delta>0$ such that $E\left(u_{\alpha_{k}}, B_{\delta}^{\Sigma}\left(\tilde{x}_{0}\right)\right)<\epsilon_{0}$. Applying Theorem 2.1, $\left\{u_{\alpha_{k}}\right\}$ converges smoothly on any $\Omega \Subset \Sigma \backslash \mathscr{Y}$. The limit map is a harmonic map from $\Sigma \backslash \mathscr{Y}$ into $N$. Theorem 2.2 tells us that the singular points of the limit map can be removed, in other words, it is a harmonic map from $\Sigma$ into $N$.

If $x_{0} \in \mathscr{Y}$, it is easy to check that

$$
\left\|\nabla u_{\alpha_{k}}\right\|_{C^{0}\left(B_{t}^{\Sigma}\left(x_{0}\right)\right)} \rightarrow+\infty
$$

for any $t$. Choose $x_{\alpha_{k}} \in B_{\delta}^{\Sigma}\left(x_{0}\right)$ such that

and let

$$
\left|\nabla u_{\alpha_{k}}\left(x_{\alpha_{k}}\right)\right|=\max _{B_{\delta}^{\Sigma}\left(x_{0}\right)}\left|\nabla u_{\alpha_{k}}\right|
$$

$$
\lambda_{\alpha_{k}}=\frac{1}{\max _{B_{\delta}^{\Sigma}\left(x_{0}\right)}\left|\nabla u_{\alpha_{k}}\right|} .
$$

It is easy to see that $x_{\alpha_{k}} \rightarrow x_{0}$ as $k \rightarrow \infty$. Then, in an isothermal coordinate system around $x_{0}$, we may define

$$
v_{k}(x)=u_{\alpha_{k}}\left(x_{\alpha_{k}}+\lambda_{\alpha_{k}} x\right) .
$$

It is well known that $v_{k}$ converges in $C^{\infty}\left(D_{R}\right)$ to a harmonic map $v^{1}: \mathbb{C} \rightarrow N$ for any fixed $R$, where $D_{R}=D_{R}(0)=\{z:|z|<R\} \subset \mathbb{C}$ is a disk with radius $R>0$. We can regard $v^{1}$ as a harmonic map from $S^{2}$ into $N$. Usually, $v^{1}$ is called the first bubble. For the details on getting all the bubbles we refer to the appendix of [ $\mathrm{Li}$ and Wang 2010b]. Moreover, in [Li and Wang 2010a] (see also [Chen and Tian 1999; Hong and Yin 2010]) we prove the following theorem which will be used later.

Theorem 2.3. Let $(\Sigma, g)$ be a closed Riemann surface and $N$ a compact Riemannian manifold. Suppose that $H$ is a fixed homotopy class of maps from $\Sigma$ into $N$ and $u_{\alpha}$ is a minimizer of $E_{\alpha}$ in the set $W^{1,2 \alpha}(\Sigma, N) \cap H$. Then when $\alpha \rightarrow 1$ there exists a subsequence $\left\{u_{\alpha}\right\}$ and harmonic map $u_{0}$ such that $\left\{u_{\alpha}\right\}$ converges to $u_{0}$ weakly in $W^{1,2}(\Sigma, N)$ and blows up at finitely many points $\left\{p_{i}: i=1,2, \ldots, m\right\}$. Moreover, associated with each $\left\{p_{i}\right\}$ there exist finitely many harmonic maps $w_{i_{j}}$ from $S^{2}$ into $N, j=1,2, \ldots, i_{0}$, such that

$$
\lim _{\alpha \rightarrow 1} E_{\alpha}\left(u_{\alpha}\right)=E\left(u_{0}\right)+\sum_{i=1}^{m} \sum_{j=1}^{i_{0}} E\left(w_{i_{j}}\right) .
$$




\section{Construction of the counterexample}

3A. Constructing the manifold $(\boldsymbol{N}, \boldsymbol{h})$. Let $h_{1}$ be the standard metric on

$$
Y_{1}=\mathbb{T}^{3}=S^{1} \times S^{1} \times S^{1}=\mathbb{R}^{3} / 2 \pi \mathbb{Z} \oplus 2 \pi \mathbb{Z} \oplus 2 \pi \mathbb{Z} .
$$

Let $B_{r}(p)$ denote a geodesic ball in $\mathbb{T}^{3}$ with radius $r$ and center $p$. Fix a point $p \in Y_{1}$, and set

$$
X_{1}=\mathbb{T}^{3} \backslash B_{r}(p),
$$

where $r<\pi /(4 \sqrt{3}+2)$. It is easy to see that the injective radius of $Y_{1}$ at $p$ is $\pi$ and $B_{\pi}(p) \backslash B_{r}(p)$ is isometric to

$$
\mathbb{T}_{0}=\left(S^{2} \times(-\log \pi,-\log r], e^{-2 t}\left(d \mathfrak{s}^{2}+d t^{2}\right)\right),
$$

where $g_{\mathfrak{s}}=d \mathfrak{s}^{2}$ is the standard metric over $S^{2}$. It is also easy to check that $\mathbb{T}_{0}$ is isometric to

$$
\mathbb{T}_{0}^{\prime}=\left(S^{2} \times\left[0, \log \frac{\pi}{r}\right), e^{2 t+2 \log r}\left(d \mathfrak{s}^{2}+d t^{2}\right)\right)
$$

and

$$
\mathbb{T}_{0}^{\prime \prime}=\left(S^{2} \times\left(-\log \frac{\pi}{r}, 0\right], e^{-2 t+2 \log r}\left(d \mathfrak{s}^{2}+d t^{2}\right)\right) .
$$

Let $\left(X_{2}, h_{2}\right)=\left(X_{1}, h_{1}\right)$. We consider the quotient space of $X_{1} \cup X_{2}$, obtained by gluing every point $x \in \partial X_{1}$ with the same point $x \in \partial X_{2}$ together. In this way, we get a closed compact manifold $N$ and a projection map $\phi: X_{1} \cup X_{2} \rightarrow N$. We set

$$
M=\phi\left(\partial B_{r}(p)\right) .
$$

On $N \backslash M$, the metric $h_{0}=\left(\phi^{-1}\right)^{*}\left(h_{1}\right) \cup\left(\phi^{-1}\right)^{*}\left(h_{2}\right)$ is well defined and can be extended to a metric $g_{0}$ over $N$. However, $g_{0}$ is not smooth and need to be modified. Obviously, $M$ has a neighborhood which is isometric to

$$
T=\left(S^{2} \times\left(-\log \frac{\pi}{r}, \log \frac{\pi}{r}\right), e^{2|t|+2 \log r}\left(d \mathfrak{s}^{2}+d t^{2}\right)\right) .
$$

In fact, $T$ is obtained by gluing $\mathbb{T}_{0}^{\prime}$ and $\mathbb{T}_{0}^{\prime \prime}$ along $S^{2} \times\{0\}$.

We let $\psi$ be a smooth function defined on $\left(-\log \frac{\pi}{r}, \log \frac{\pi}{r}\right)$ which satisfies

(1) $\psi=e^{2|t|+2 \log r}$ when $|t| \geq \log 2$;

(2) $\psi^{\prime}<0$ on $(-\log 2,0)$ and $\psi^{\prime}>0$ on $(0, \log 2)$.

Note that (2) implies that 0 is the only critical point of $\psi$ on $(-\log 2, \log 2)$.

We define a new metric $h$ on $N$ which is $h_{0}$ on $N \backslash T$, and $\psi(t)\left(d \mathfrak{s}^{2}+d t^{2}\right)$ on $T$. It is easy to see that $h$ is smooth on $N$. For convenience, we set

$$
Q(a)=S^{2} \times\left(-\log \frac{a}{r}, \log \frac{a}{r}\right) \subset T .
$$


Obviously, we have

$$
\phi^{-1}(Q(a)) \cap X_{1}=B_{a}(p) \backslash B_{r}(p) \subset Y_{1} .
$$

Lemma 3.1. Let $(N, h), T$ and $Q(a)$ be defined as above. Assume that $u: S^{2} \rightarrow$ $(N, h)$ is a nontrivial harmonic map with $u\left(S^{2}\right) \subset Q(\pi)=T$. Then $u$ is a harmonic map from $S^{2}$ into $M$.

Proof. Let $u=(v, f): S^{2} \rightarrow Q(\pi)$ be a harmonic map, where $v \in C^{\infty}\left(S^{2}, S^{2}\right)$ and $f \in C^{\infty}\left(S^{2}\right)$. The energy can be written as

$$
E(u)=\frac{1}{2} \int_{S^{2}}|\nabla u|^{2} d V=\frac{1}{2} \int_{S^{2}}\left(|\nabla v|^{2}+|\nabla f|^{2}\right) \psi(f) d V .
$$

Here $d V=d V_{g_{\mathfrak{s}}}$ is the standard volume form of $S^{2}$. By a direct calculation, it is easy to see that $u$ satisfies

$$
\begin{aligned}
-\nabla(\psi(f) \nabla v)+\psi(f)|\nabla v|^{2} v & =0, \\
-\nabla(\psi(f) \nabla f)+\frac{1}{2}\left(|\nabla v|^{2}+|\nabla f|^{2}\right) \psi^{\prime}(f) & =0 .
\end{aligned}
$$

Multiplying both sides of the second equation of (3-1) by $f$ and then integrating the obtained identity over $S^{2}$, we get the identity

$$
\int_{S^{2}}\left(|\nabla f|^{2} \psi(f)+\frac{1}{2}\left(|\nabla v|^{2}+|\nabla f|^{2}\right) \psi^{\prime}(f) f\right) d V=0 .
$$

Noting that $\psi^{\prime}(f) f \geq 0$ always holds true, we infer from the above identity

$$
\int_{S^{2}}|\nabla f|^{2} \psi(f) d \mathfrak{s}=\frac{1}{2} \int_{S^{2}}\left(|\nabla v|^{2}+|\nabla f|^{2}\right) \psi^{\prime}(f) f d V=0 .
$$

This implies that $\nabla f=0$ and $f$ is a constant. Moreover, from the above identity we also have

$$
|\nabla v|^{2} \psi^{\prime} f \equiv 0
$$

Since $u$ is nontrivial by assumption, there always exists a point $x_{1} \in S^{2}$ such that $|\nabla v|\left(x_{1}\right) \neq 0$. Hence we conclude that $\psi^{\prime}(f) f \equiv 0$ which implies $f \equiv 0$. It follows that $v$ is a harmonic map from $S^{2}$ into $M$.

Lemma 3.2. Let $(N, h)$ and $Q$ be the same as in Lemma 3.1. Assume that $u$ is a harmonic map from $S^{2}$ into $(N, h)$ such that $u\left(S^{2}\right) \cap Q(2 r) \neq \varnothing$ and $u\left(S^{2}\right) \cap$ $\partial Q(\pi) \neq \varnothing$. Then we have

$$
E(u) \geq \pi(\pi-2 r)^{2} .
$$


Proof. Without loss of generality, we assume $p_{1} \in X_{1}$ is such that $p_{1}$ is in $\partial B_{\pi}(p)$ in $Y_{1}$ and $\phi\left(p_{1}\right)$ is in $u\left(S^{2}\right)$. First, $u$ is a branched minimal surface since $u$ is a harmonic map from $S^{2}$ into $N$. On the other hand, as $h$ is flat on $\phi\left(B_{\pi-2 r}\left(p_{1}\right)\right)$, it is easy to check that $u\left(S^{2}\right) \cap \phi\left(B_{\pi-2 r}\left(p_{1}\right)\right)$ is a stationary varifold. Denote by $\mu\left(u\left(S^{2}\right) \cap B_{\pi-2 r}\left(p_{1}\right)\right)$ the area of $u\left(S^{2}\right) \cap B_{\pi-2 r}\left(p_{1}\right)$. By the monotonicity inequality for stationary varifolds (see [Simon 1983]), we have

$$
\frac{\mu\left(u\left(S^{2}\right) \cap B_{\pi-2 r}\left(p_{1}\right)\right)}{\pi(\pi-2 r)^{2}} \geq 1 .
$$

In light of this inequality and the fact $E(u) \geq \mu\left(u\left(S^{2}\right) \cap B_{\pi-2 r}\left(p_{1}\right)\right)$, we derive the desired inequality

$$
E(u) \geq \pi(\pi-2 r)^{2}
$$

and the proof is complete.

Since $h$ is flat on $N \backslash Q(2 r)$, we have the following lemma.

Lemma 3.3. Let $(N, h)$ and $Q$ be the same as in Lemma 3.1. Then there is no nontrivial harmonic map $u: S^{2} \rightarrow(N, h)$ such that $u\left(S^{2}\right) \cap \overline{Q(2 r)}=\varnothing$.

By the definition of $\psi$, it is easy to check that

$$
4 \pi \psi(0) \leq 16 \pi r^{2}<\frac{1}{3} \pi(\pi-2 r)^{2}
$$

when $r$ is small enough. Using Lemma 3.2 and Lemma 3.3, we get the following result.

Corollary 3.4. Let $(N, h)$ and $Q$ be the same as in Lemma 3.1. Assume that $u$ is a nontrivial harmonic map with $E(u)<\pi(\pi-2 r)^{2}$; then

$$
E(u)=4 m \pi \psi(0)
$$

where $m$ is a positive integer.

It is easy to check that

$$
12 \pi \psi(0)<48 \pi r^{2}<\pi(\pi-2 r)^{2},
$$

if $r<\frac{\pi}{4 \sqrt{3}+2}$. Therefore we know that if $E(u)<12 \pi \psi(0)$ and $u$ is a nontrivial harmonic map, then $E(u)=4 \pi \psi(0)$ or $8 \pi \psi(0)$.

3B. The homotopy class $\left[\boldsymbol{u}_{\boldsymbol{k}}\right]$. We have $\pi_{1}\left(Y_{1}\right)=\pi_{1}\left(\mathbb{T}^{3}\right)=\mathbb{Z}^{3}$. Let $\beta \in \pi_{1}\left(Y_{1}\right)$ which represents $(1,0,0)$. Let $x_{1}, x_{2} \in M$, and $\gamma_{0}$ be a curve in $M$ such that $\gamma_{0}(0)=$ $x_{2}$, and $\gamma_{0}(1)=x_{1}$. Let $\gamma_{k}:[0,1] \rightarrow X$ be a curve with $\gamma_{k}(0)=x_{1}, \gamma_{k}(1)=x_{2}$ and $\left[\gamma_{k}+\gamma_{0}\right]=k \beta$. Let $w_{0}$ be a diffeomorphism from $S^{2}$ onto $M$ satisfying $w_{0}(0,0,1)=x_{1}$ and $w_{0}(0,0,-1)=x_{2}$, where $(0,0,1)$ and $(0,0,-1)$ are the north and the south poles of $S^{2} \subset \mathbb{R}^{3}$, respectively. 
For the sake of convenience, we introduce the stereographic projection coordinates on $S^{2}$ with the south pole corresponding to $\infty$. Thus, $w_{0}: S^{2} \rightarrow N$ can be viewed as a map from $\mathbb{C} \cup\{\infty\}$ into $N$. For simplicity, we neglect the stereographic projection map $\mathfrak{S}: S^{2} \rightarrow \mathbb{C} \cup\{\infty\}$ and still denote $w_{0} \circ \mathfrak{S}^{-1}$ by $w_{0}$.

By the continuity of $w_{0}$, there exists a small $\delta_{0}>0$ such that $w_{0}\left(D_{\delta_{0}}\right)$ is contained in a small neighborhood of $x_{1}$, where $D_{\delta_{0}}=\left\{z \in \mathbb{C}:|z|<\delta_{0}\right\}$, and a large $R_{0}>0$ such that $w_{0}\left(\mathbb{C} \backslash D_{R_{0}}\right)$ is contained in a small neighborhood of $x_{2}$, where $D_{R_{0}}=\left\{z \in \mathbb{C}:|z|<R_{0}\right\}$.

In order to construct a sequence of maps, we need to define the following two smooth nonnegative functions $\lambda$ and $v$ on $[0, \infty)$ :

(1) $\lambda(s):[0, \infty] \rightarrow[0,1]$ with $\lambda(s) \equiv 0$ as $s \in\left[0, \delta_{0}\right]$ and $\lambda(s) \equiv 1$ as $s \in\left[2 \delta_{0}, \infty\right)$.

(2) $v(s):[0, \infty) \rightarrow[0,1]$ with $v(s) \equiv 1$ as $s \in\left[0, R_{0}-R_{0}^{c}\right]$ and $v(s) \equiv 0$ as $s>R_{0}$, where $R_{0}^{c}$ is a small positive constant number.

Now we define a sequence of maps $u_{k}: S^{2} \rightarrow N$ by

$$
u_{k}= \begin{cases}w_{0}(\lambda(|z|) z) & |z| \geq \delta_{0}, \\ \gamma_{k}\left(\frac{\log |z|-\log R_{0} \epsilon_{0}}{\log \delta_{0}-\log R_{0} \epsilon_{0}}\right) & R_{0} \epsilon_{0}<|z|<\delta_{0}, \\ w_{0}\left(\frac{z}{v\left(|z| / \epsilon_{0}\right) \epsilon_{0}}\right) & |z| \leq \epsilon_{0} R_{0} .\end{cases}
$$

Here $\epsilon_{0}>0$ is a fixed constant number such that $R_{0} \epsilon_{0}<\delta_{0}$. By the arguments in [Li and Wang 2010b], for any $i \neq j, u_{i}$ is not homotopic to $u_{j}$. For the sequence $\left\{u_{i}\right\}$ constructed above, we have the following lemma:

Lemma 3.5. Let $u_{k}$ be the maps from $S^{2}$ into $(N, h)$ constructed above and $\left[u_{k}\right]$ denote the class of maps in $W^{1,2}\left(S^{2}, N\right) \cap C\left(S^{2}, N\right)$, each map of which is homotopic to $u_{k}$. For any fixed $k$, we have

$$
\inf _{u \in\left[u_{k}\right]} E(u)=8 \pi \psi(0) .
$$

Moreover, $\inf E(u)$ cannot be attained by a harmonic map belonging to $\left[u_{k}\right]$.

Proof. First of all, we prove that for every fixed $k$

$$
\inf _{u \in\left[u_{k}\right]} E(u) \leq 8 \pi \psi(0) .
$$

Denote $z_{1}=(0,0,1)$ and $z_{2}=(0,0,-1) \in S^{2}$. Without loss of generality, we assume $w_{0}$ is a harmonic map from $S^{2}$ into $M$ with $E\left(w_{0}\right)=4 \pi \psi(0)$ with $w_{0}\left(z_{1}\right)=x_{1}$ and $w_{0}\left(z_{2}\right)=x_{2}$. Let $\mathfrak{S}$ be the stereographic projection from $S^{2} \backslash\left\{z_{2}\right\}$ to $\mathbb{C}$ and

$$
\hat{u}_{0}(z)=w_{0}\left(\mathfrak{S}^{-1}(z)\right): \mathbb{C} \cup\{\infty\} \rightarrow N
$$


Choose a coordinate system $\left(y_{1}, y_{2}, y_{3}\right)$ in a geodesic ball $B_{\rho}\left(x_{1}\right)$ around $x_{1} \in N$ with $x_{1}=(0,0,0)$ and $\left\{\left(y_{1}, y_{2}, 0\right):\left(y_{1}, y_{2}, 0\right) \in B_{\rho}\left(x_{1}\right)\right\} \subset M$. By the continuity of $w_{0}$, there exists a small $\delta>0$ such that $w_{0}(z) \in B_{\rho}\left(x_{1}\right)$ when $|z|<\delta$. We define

$$
u_{0}^{\prime}=\eta_{1} \hat{u}_{0}
$$

where $\eta_{1}$ is a smooth nonnegative function which equals 1 outside $D_{2 \delta}, 0$ on $D_{\delta}$, and satisfies $\left|\nabla \eta_{1}\right|<\frac{C}{\delta}$. Here $D_{2 \delta} \subset \mathbb{C}$ denotes the disk centered at the origin. Then we have

$$
\int_{D_{2 \delta}}\left|\nabla u_{0}^{\prime}\right|^{2} d x^{2} \leq 2 \int_{D_{2 \delta}}\left(\left|\nabla \eta_{1}\right|^{2}\left|\hat{u}_{0}\right|^{2}+\left|\nabla \hat{u}_{0}\right|^{2}\right) d x^{2} \leq C \delta
$$

Thus $u_{0}^{\prime}$ satisfies

$$
\operatorname{dist}^{M}\left(u_{0}^{\prime}, \hat{u}_{0}\right)<C \delta, \quad E\left(u_{0}^{\prime}\right)<4 \pi \psi(0)+C \delta, \quad \text { and } \quad u_{0}^{\prime}\left(D_{\delta}\right)=x_{1} .
$$

Since $E$ is conformally invariant, $\hat{u}_{0}(1 / z)$ is also a harmonic map from $\mathbb{C} \backslash\{0\}$ into $N$ with

$$
E\left(\hat{u}_{0}(1 / z), \mathbb{C}\right)=E\left(\hat{u}_{0}(z), \mathbb{C}\right) .
$$

Thus, $\hat{u}_{0}(1 / z)$ can be extended smoothly to $\{0\}$. Choose a coordinate system $\left(y_{1}, y_{2}, y_{3}\right)$ in a geodesic ball $B_{\rho}\left(x_{2}\right)$ around $x_{2} \in N$ with $x_{2}=(0,0,0)$ and $\left\{\left(y_{1}, y_{2}, 0\right):\left(y_{1}, y_{2}, 0\right) \in B_{\rho}\left(x_{2}\right)\right\} \subset M$. By the continuity of $w_{0}$, there exists a large $R>0$ such that $\hat{u}_{0}(z) \in B_{\rho}\left(x_{2}\right)$ as $|z|>R$. Then we have

$$
\hat{u}_{0}(1 / z)=O(z) \text { and }\left|\nabla \hat{u}_{0}(1 / z)\right|=O(1), \quad \text { as } z \rightarrow 0 .
$$

Hence, we have

$$
\hat{u}_{0}(z)=O(1 / z) \text { and } \quad\left|z^{2} \nabla \hat{u}_{0}(z)\right|=O(1), \quad \text { as } z \rightarrow \infty .
$$

Let

$$
u_{0}^{\prime \prime}(z)=\eta_{2}(|z|) \hat{u}_{0}(z),
$$

where $\eta_{2}(|z|)$ is a smooth nonnegative function which equals 0 outside $D_{R}, 1$ on $D_{R / 2}$, and satisfies $\left|\nabla \eta_{1}\right|<\frac{C}{R}$. Then we have

$$
\int_{\mathbb{C} \backslash D_{R / 2}}\left|\nabla u_{0}^{\prime \prime}\right|^{2} d x^{2} \leq 2 \int_{D_{R} \backslash D_{R / 2}}\left(\left|\nabla \eta_{2}\right|^{2}\left|\hat{u}_{0}\right|^{2}+\left|\nabla \hat{u}_{0}\right|^{2}\right) d x^{2} \leq \frac{C}{R} .
$$

Thus

$$
\operatorname{dist}^{M}\left(u_{0}^{\prime \prime}, u_{0}\right)<\frac{C}{R}, \quad E\left(u_{0}^{\prime \prime}\right)<4 \pi \psi(0)+\frac{C}{R}, \quad \text { and } \quad u_{0}^{\prime \prime}\left(\mathbb{C} \backslash D_{R}\right)=x_{2} .
$$


We define

$$
\phi_{k}= \begin{cases}u_{0}^{\prime}(z), & |z| \geq \delta, \\ \gamma_{k}\left(\frac{\log |z|-\log R \epsilon}{\log \delta-\log R \epsilon}\right), & R \epsilon<|z|<\delta, \\ u_{0}^{\prime \prime}\left(\frac{z}{\epsilon}\right), & |z| \leq \epsilon R .\end{cases}
$$

By a direct calculation, we obtain

$$
\begin{aligned}
\int_{D_{\delta} \backslash D_{R \epsilon}}\left|\nabla \phi_{k}\right|^{2} & =2 \pi \int_{R \epsilon}^{\delta}\left|\frac{\partial \gamma_{k}}{\partial r}\right|^{2} r d r \\
& <\frac{c\left\|\dot{\gamma}_{k}\right\|_{L^{\infty}}^{2}}{(-\log R \epsilon+\log \delta)^{2}} \int_{R \epsilon}^{\delta} \frac{d r}{r}=\frac{c\left\|\dot{\gamma}_{k}\right\|_{L^{\infty}}^{2}}{\log \delta-\log R \epsilon} .
\end{aligned}
$$

Thus, for any $\epsilon_{1}>0$, we can choose suitable $\delta, R$ and $\epsilon$ such that

$$
E\left(\phi_{k}\right)<8 \pi \psi(0)+\epsilon_{1} .
$$

Obviously, $\varphi_{k}=\phi_{k}\left(\mathfrak{S}^{-1}\right)$ is homotopic to $u_{k}$, denoted by $\varphi_{k} \sim u_{k}$. Thus, we get (3-2).

Next, we prove that $\inf _{u \in\left[u_{k}\right]} E(u)$ cannot be attained by a harmonic map. Assume it is attained by a harmonic map $v_{0}$. Recall that

$$
8 \pi \psi(0)<12 \pi \psi(0)<48 \pi r^{2}<\pi(\pi-2 r)^{2},
$$

where $r>0$ is small enough. By Lemma 3.2, $v_{0}\left(S^{2}\right) \subset Q(\pi)$. Thus $v_{0}$ is a harmonic map from $S^{2}$ into $M$. This contradicts the fact $v_{0} \sim u_{k}$. Hence $\inf _{u \in\left[u_{k}\right]} E(u)$ cannot be attained by a harmonic map.

Let $u_{\alpha}$ be the $\alpha$-harmonic map such that, for fixed $k$,

$$
E_{\alpha}\left(u_{\alpha}\right)=\inf _{u \in\left[u_{k}\right] \cap W^{1,2 \alpha}\left(S^{2}, N\right)} E_{\alpha}(u) .
$$

Then each map of $\left\{u_{\alpha}\right\}$ is minimizing and belongs to $\left[u_{k}\right]$. We claim that $\left\{u_{\alpha}\right\}$ does not converge smoothly. Otherwise, the limit map is a harmonic map from $S^{2}$ into $N$, which is homotopic to $u_{k}$. This contradicts the above fact that inf $u_{u \in\left[u_{k}\right]} E(u)$ cannot be attained by a harmonic map. Hence, the bubbles must appear in the convergence of $u_{\alpha}$. If we denote the weak limit of $\left\{u_{\alpha}\right\}$ as $u_{0}$ and the bubbles as $v^{1}, \ldots, v^{m}$, then, by Theorem 2.3, we have

$$
\inf _{u \in\left[u_{k}\right]} E(u)=\lim _{\alpha \rightarrow 1} E_{\alpha}\left(u_{\alpha}\right)=E\left(u_{0}\right)+\sum_{i=1}^{m} E\left(v^{i}\right) .
$$

Since $E\left(u_{0}\right)$ and $E\left(v^{i}\right)$ are smaller than $\pi(\pi-2 r)^{2}, E\left(u_{0}\right)+\sum_{i=1}^{m} E\left(v^{i}\right)$ can only equal $8 \pi \psi(0)$ or $4 \pi \psi(0)$. 
Next, we will show that the following identity does not hold true:

$$
E\left(u_{0}\right)+\sum_{i=1}^{m} E\left(v^{i}\right)=4 \pi \psi(0) .
$$

If we assume this is true, then $u_{0}$ is trivial and $u_{\alpha}$ has only one bubble $v^{1}$. To derive a contradiction, we only need to prove $u_{\alpha} \sim v^{1}$.

Let $x_{0} \in S^{2}$ be a blowup point. Take an isothermal coordinate system around $x_{0}$ with $x_{0}=(0,0)$ on $S^{2}=\mathbb{C} \cup\{\infty\}$. Let $v^{1}$ be the limit map of $u_{\alpha}\left(z_{\alpha}+\lambda_{\alpha}^{1} z\right)$, where $z_{\alpha} \rightarrow 0, \lambda_{\alpha}^{1} \rightarrow 0$. Then

$$
v_{\alpha}^{1}(z)=u_{\alpha}\left(z_{\alpha}+\lambda_{\alpha}^{1} z\right)
$$

converges smoothly to $v^{1}$ on any $D_{R}=D_{R}(0) \subset \mathbb{C}$. Moreover, $u_{\alpha}$ converges smoothly in $\mathbb{C} \cup\{\infty\} \backslash D_{1 / R}$ to a point $y_{0} \in N$. For us to prove $u_{\alpha} \sim v^{1}$, it is enough to check that for any $\epsilon>0$, there exists an $R>0$, such that

$$
\sup _{t \in\left[R \lambda_{\alpha}^{1}, 1 / R\right]} \operatorname{osc}_{\partial D_{t}\left(z_{\alpha}\right)} u_{\alpha}<\epsilon .
$$

Indeed, if this is not true then there exists a sequence of $\lambda_{\alpha}^{2}$ with $\lambda_{\alpha}^{2} \rightarrow 0$ and $\lambda_{\alpha}^{2} / \lambda_{\alpha}^{1} \rightarrow+\infty$, such that

$$
\operatorname{osc}_{\partial D_{\lambda_{\alpha}^{2}}\left(z_{\alpha}\right)} u_{\alpha} \rightarrow \epsilon_{1} \neq 0
$$

Let

$$
v_{\alpha}^{2}(z)=u_{\alpha}\left(z_{\alpha}+\lambda_{\alpha}^{2} z\right) .
$$

If the sequence $\left\{v_{\alpha}^{2}\right\}$ has blowup points, then at each blowup point there exists at least a bubble of $\left\{v_{\alpha}^{2}\right\}$ which is also a bubble of $u_{\alpha}$ and is different from the previous bubble $v^{1}$. However, this is impossible since there only exists one bubble for $\left\{u_{\alpha}\right\}$. Hence, we infer that as $\alpha \rightarrow 1,\left\{v_{\alpha}^{2}\right\}$ converges smoothly on $D_{R^{\prime}} \backslash D_{1 / R^{\prime}} \subset \mathbb{C}$ for any $R^{\prime}$. It follows that

$$
\operatorname{osc}_{\partial D_{1}} v_{\alpha}^{2} \rightarrow \epsilon_{1} \neq 0
$$

This means that the limit map of $\left\{v_{\alpha}^{2}\right\}$ is not trivial and the limit map is also a bubble of $\left\{u_{\alpha}\right\}$ which is different from $v^{1}$. This is a contradiction. Thus, we conclude

$$
\inf _{u \in\left[u_{k}\right]} E(u)=E\left(u_{0}\right)+\sum_{i=1}^{m} E\left(v^{i}\right)=8 \pi \psi(0) .
$$

This completes the proof of the lemma.

By the Sobolev embedding theorem, we know that for $\alpha>1$,

$$
W^{1,2 \alpha}\left(S^{2}, N\right) \subset C\left(S^{2}, N\right) .
$$


For simplicity, let $\left[u_{k}\right]^{\alpha}$ denote the class of maps belonging to $W^{1,2 \alpha}\left(S^{2}, N\right)$, each map of which is homotopic to $u_{k}$. In fact, it is easy to see that

$$
\left[u_{k}\right]^{\alpha}=\left[u_{k}\right] \cap W^{1,2 \alpha}\left(S^{2}, N\right) .
$$

From now on, we will always denote the smooth map which attains $\inf \tilde{u}_{u \in\left[u_{k}\right]^{\alpha}} E_{\alpha}(u)$ by $u_{\alpha, k}$ :

$$
E_{\alpha}\left(u_{\alpha, k}\right)=\inf _{u \in\left[u_{k}\right]^{\alpha}} E_{\alpha}(u)
$$

Lemma 3.6. For any $\lambda_{0}>8 \pi \psi(0)$, there exists a sequence $\left\{\alpha_{k}\right\}$ with $\alpha_{k} \rightarrow 1$ and a sequence $\left\{i_{k}\right\}$ such that $E_{\alpha_{k}}\left(u_{\alpha_{k}, i_{k}}\right)=\lambda_{0}$ for every $k$.

Proof. For $\alpha \in\left[1, \alpha_{0}\right)$ where $\alpha_{0}-1>0$ is small enough, we define the following function

$$
\varphi_{k}(\alpha)=\inf _{u \in\left[u_{k}\right]^{\alpha}} E_{\alpha}(u) .
$$

Firstly, we need to show that for any fixed $\alpha \in\left(1, \alpha_{0}\right)$,

$$
\lim _{k \rightarrow+\infty} \varphi_{k}(\alpha)=+\infty .
$$

If this is false, then there exists a constant $C$ such that $\varphi_{k}(\alpha) \leq C$ as $k$ is large enough. We note that for any small $\delta$ and $x \in S^{2}$,

$$
E\left(u_{\alpha, k}, B_{\delta}(x)\right)=\frac{1}{2} \int_{B_{\delta}(x)}\left|\nabla u_{\alpha, k}\right|^{2} \leq \frac{1}{2}\left(\int_{B_{\delta}(x)}\left|\nabla u_{\alpha, k}\right|^{2 \alpha}\right)^{1 / \alpha}\left|B_{\delta}(x)\right|^{(\alpha-1) / \alpha} .
$$

Hence, we can pick a fixed $\delta$, which is small enough, such that

$$
E\left(u_{\alpha, k}, B_{\delta}(x)\right)<\epsilon_{0} .
$$

Thus, by Theorem 2.1, there exists a subsequence of $u_{\alpha, k}$ which converges smoothly to a smooth map $u_{0}$ as $k$ tends to $\infty$. Hence, we know that $u_{\alpha, k}$ are homotopic to $u_{0}$ for any $k$. This contradicts the fact that $u_{\alpha, i}$ is not homotopic to $u_{\alpha, j}$ as $i \neq j$.

Next, we want to prove $\varphi_{k}$ is continuous on $\left[1, \alpha_{0}\right)$. Using (3-4) again, we can prove that, for a fixed small $\epsilon>0$,

$$
\left\|\nabla u_{\alpha, k}\right\|_{C^{0}\left(S^{2}\right)}<\Lambda(\epsilon)
$$

for any $\alpha \in\left(1+\epsilon, \alpha_{0}\right)$. For any $\alpha, \alpha^{\prime} \in\left(1+\epsilon, \alpha_{0}\right)$, we have

$$
\begin{aligned}
\varphi_{k}(\alpha) & \geq \frac{1}{2}\left(1+C_{1}^{2}\right)^{\alpha-\alpha^{\prime}} \int_{S^{2}}\left(1+\left|\nabla u_{\alpha, k}\right|^{2}\right)^{\alpha^{\prime}}-\frac{1}{2} \\
& \geq\left(1+C_{1}^{2}\right)^{\alpha-\alpha^{\prime}} \varphi_{k}\left(\alpha^{\prime}\right)+\frac{1}{2}\left(1+C_{1}^{2}\right)^{\alpha-\alpha^{\prime}}-\frac{1}{2},
\end{aligned}
$$


where

$$
C_{1}= \begin{cases}0 & \text { when } \alpha>\alpha^{\prime} \\ \Lambda(\epsilon) & \text { when } \alpha<\alpha^{\prime}\end{cases}
$$

It follows that

$$
\varliminf_{\alpha \rightarrow \alpha^{\prime}} \varphi_{k}(\alpha) \geq \varphi_{k}\left(\alpha^{\prime}\right) .
$$

On the other hand, we also have

$$
\varphi_{k}\left(\alpha^{\prime}\right) \geq \frac{1}{2}\left(1+C_{2}^{2}\right)^{\alpha^{\prime}-\alpha} \int_{S^{2}}\left(1+\left|\nabla u_{\alpha^{\prime}, k}\right|^{2}\right)^{\alpha}-\frac{1}{2},
$$

where

$$
C_{2}= \begin{cases}0 & \text { when } \alpha^{\prime}>\alpha, \\ \left\|\nabla u_{\alpha^{\prime} k}\right\|_{L^{\infty}} & \text { when } \alpha^{\prime}<\alpha .\end{cases}
$$

It follows that

$$
\varphi_{k}\left(\alpha^{\prime}\right) \geq\left(1+C_{2}^{2}\right)^{\alpha^{\prime}-\alpha} \varphi_{k}(\alpha)+\frac{1}{2}\left(1+C_{2}^{2}\right)^{\alpha^{\prime}-\alpha}-\frac{1}{2},
$$

and

$$
\varlimsup_{\alpha \rightarrow \alpha^{\prime}} \varphi_{k}(\alpha) \leq \varphi_{k}\left(\alpha^{\prime}\right)
$$

Therefore, we have

$$
\lim _{\alpha \rightarrow \alpha^{\prime}} \varphi_{k}(\alpha)=\varphi_{k}\left(\alpha^{\prime}\right),
$$

and we have shown the continuity of $\varphi_{k}(\alpha)$ on $\left(1, \alpha_{0}\right)$.

Next, we want to prove that $\varphi_{k}(\alpha)$ is left continuous at 1 . Equivalently, we need to show

$$
\lim _{\alpha \searrow 1} \varphi_{k}(\alpha)=\varphi_{k}(1)
$$

Obviously, for any fixed $u \in W^{1,2}\left(S^{2}, N\right)$ and $\alpha_{1}>\alpha_{2}>1$,

$$
E_{\alpha_{1}}(u) \geq E_{\alpha_{2}}(u) \geq E(u) .
$$

It follows that

$$
\varphi_{k}\left(\alpha_{1}\right) \geq \varphi_{k}\left(\alpha_{2}\right) \geq \varphi_{k}(1) .
$$

Hence, $\lim _{\alpha \searrow 1} \varphi_{k}$ exists and

$$
\lim _{\alpha \searrow 1} \varphi_{k}(\alpha) \geq \varphi_{k}(1)
$$

On the other hand, note that $u_{k}$ is a smooth map. Then for any $\epsilon>0$, there exists a smooth map $u_{k}^{\prime} \in C^{\infty}\left(S^{2}, N\right)$ which is homotopic to $u_{k}$ (i.e., $u_{k}^{\prime} \sim u_{k}$ ), and satisfies

$$
E\left(u_{k}^{\prime}\right) \leq \varphi_{k}(1)+\epsilon
$$


Since

$$
\lim _{\alpha \searrow 1} E_{\alpha}\left(u_{k}^{\prime}\right)=E\left(u_{k}^{\prime}\right) \text { and } \varphi_{k}(\alpha) \leq E_{\alpha}\left(u_{k}^{\prime}\right)
$$

we have

$$
\lim _{\alpha \searrow 1} \varphi_{k}(\alpha) \leq \varphi_{k}(1)+\epsilon,
$$

which implies (3-5), and shows that $\varphi_{k}(\alpha)$ is continuous on $\left[1, \alpha_{0}\right)$ for any fixed $k$.

By (3-3), for any given sequence $\left\{\alpha_{k}^{\prime}\right\}$ with $\alpha_{k}^{\prime} \rightarrow 1$, there exists a sequence $\left\{i_{k}\right\}$ such that $E_{\alpha_{k}^{\prime}}\left(u_{\alpha_{k}^{\prime}, i_{k}}\right)>\lambda_{0}$, or equivalently, $\varphi_{i_{k}}\left(\alpha_{k}^{\prime}\right)>\lambda_{0}$. Lemma 3.5 tells us that $\varphi_{i_{k}}(1)=8 \pi \psi(0)$ for any $i_{k}$. By the assumption $\lambda_{0}>8 \pi \psi(0)$ we have

$$
\varphi_{i_{k}}\left(\alpha_{k}^{\prime}\right)>\lambda_{0}>\varphi_{i_{k}}(1) \text {. }
$$

Since $\varphi_{k}(\alpha)$ is continuous on $\left[1, \alpha_{0}\right)$, we conclude that for any fixed $i_{k}$ there exists $\alpha_{k} \in\left(1, \alpha_{k}^{\prime}\right)$ such that

$$
\varphi_{i_{k}}\left(\alpha_{k}\right)=E_{\alpha_{k}}\left(u_{\alpha_{k}, i_{k}}\right)=\lambda_{0} .
$$

This completes the proof.

3C. The counterexample. By Lemma 3.6, for given $\tau \in(8 \pi \psi(0), 12 \pi \psi(0))$ there exist a sequence $\left\{\alpha_{k}: \alpha_{k}>1, k \in \mathbb{N}\right\}$ with $\alpha_{k} \rightarrow 1$ and a sequence of minimizing $\alpha_{k}$-harmonic maps $v_{k} \in W^{1,2 \alpha_{k}}\left(S^{2}, N\right)$ with $v_{k} \sim u_{i_{k}}$ such that

$$
\tau=E_{\alpha_{k}}\left(v_{k}\right)=\inf _{u \in\left[u_{i_{k}}\right]^{\alpha_{k}}} E_{\alpha_{k}}(u) \text { for all } k \in \mathbb{N} .
$$

Since $v_{i}$ and $v_{j}$ are not in the same homotopy class for any $i \neq j, v_{k}$ must blow up as $k \rightarrow+\infty$. Let $v^{0}$ be the weak limit of $\left\{v_{k}\right\}$ in $W^{1,2}\left(S^{2}, N\right)$, and $v^{1}, \ldots, v^{m}$ be all the bubbles produced in the convergence of $\left\{v_{k}\right\}$. Since $E\left(v^{i}\right)<12 \pi \psi(0)$, it follows from Corollary 3.4 that $E\left(v^{i}\right)=4 \pi \psi(0)$ or $8 \pi \psi(0)$. Hence,

$$
\frac{1}{4 \pi \psi(0)}\left(E\left(v^{0}\right)+\sum_{i=1}^{m} E\left(v^{i}\right)\right)
$$

is always an integer. However, certainly $\frac{\tau}{4 \pi \psi(0)}$ is not an integer by the previous assumption. So the energy identity is not true for the sequence $\left\{v_{k}\right\}$ :

$$
\lim _{k \rightarrow \infty} E_{\alpha_{k}}\left(v_{k}\right) \neq E\left(v^{0}\right)+\sum_{i=1}^{m} E\left(v^{i}\right) .
$$

Remark 3.7. By an argument in [Li and Wang 2010b], we also have

$$
\lim _{k \rightarrow \infty} E\left(v_{k}\right) \neq E\left(v^{0}\right)+\sum_{i=1}^{m} E\left(v^{i}\right) .
$$




\section{An example of a manifold whose energy set is nondiscrete for harmonic 2-spheres}

In this section, we will construct a Riemannian manifold $(N, h)$ for which $\mathscr{E}(N, h)$ is not discrete. In other words, $\mathscr{E}(N, h)$ admits limit points.

Let $\psi(t)$ be a smooth positive function defined on $(-1,1)$ satisfying

$$
\psi(t)=e^{-1 / t^{2}} \sin \frac{1}{t}+1, \quad t \in\left(-\frac{1}{2}, \frac{1}{2}\right) .
$$

It is easy to check that the critical point of $\psi(t)$ satisfies the equation

$$
\tan \frac{1}{t}=\frac{t}{2}
$$

Thus, we can find $t_{k} \rightarrow 0$, such that $\psi^{\prime}\left(t_{k}\right)=0, \psi\left(t_{k}\right) \neq 1$ and $\psi\left(t_{k}\right) \rightarrow 1$.

Let

$$
h=\psi(t)\left(d \mathfrak{s}^{2}+d t^{2}\right),
$$

which is a metric over $S^{2} \times(-1,1)$. Let $v$ be the identity map from $S^{2}$ to $S^{2}$ and

$$
u_{k}=\left(v, t_{k}\right): S^{2} \rightarrow(N, h) \equiv\left(S^{2} \times(-1,1), h\right) .
$$

By (3-1), it is easy to see that $u_{k}$ is a harmonic map from $S^{2}$ into $\left(S^{2} \times(-1,1), h\right)$ with

$$
E\left(u_{k}\right)=4 \pi \psi\left(t_{k}\right) .
$$

Thus, $4 \pi$ is not a discrete number in $\mathscr{E}\left(S^{2} \times(-1,1), h\right)$.

\section{Acknowledgement}

The authors are grateful to thank Professor Weiyue Ding for his encouragement and beneficial suggestions. The first author is supported by NSFC, Grant No. 11131007; the second author is supported by NSFC, Grant No. 10990013.

\section{References}

[Anand 1995] C. K. Anand, "Uniton bundles", Comm. Anal. Geom. 3:3-4 (1995), 371-419. MR 96k: $58052 \mathrm{Zbl} 0848.58013$

[Chen and Tian 1999] J. Chen and G. Tian, "Compactification of moduli space of harmonic mappings", Comment. Math. Helv. 74:2 (1999), 201-237. MR 2001k:58024 Zbl 0958.53047

[Ding 1998] W.-Y. Ding, "Lectures on the heat flow of harmonic maps", preprint, National Tsing Hua University, Taiwan, 1998, Available at http://www.math.pku.edu.cn:8000/var/teacher_writings/ 20080327073216.pdf.

[Ding and Tian 1995] W.-Y. Ding and G. Tian, "Energy identity for a class of approximate harmonic maps from surfaces”, Comm. Anal. Geom. 3:3-4 (1995), 543-554. MR 97e:58055 Zbl 0855.58016

[Dong 2002] Y. Dong, “On energy gap of unitons”, Math. Z. 240:4 (2002), 677-688. MR 2003e: 
[Duzaar and Kuwert 1998] F. Duzaar and E. Kuwert, "Minimization of conformally invariant energies in homotopy classes", Calc. Var. Partial Differential Equations 6:4 (1998), 285-313. MR 99d:58045 Zbl 0909.49008

[Hong and Yin 2010] M. Hong and H. Yin, "On the Sacks-Uhlenbeck flow of Riemannian surfaces", preprint, 2010. arXiv 1007.3052

[Jost 1991] J. Jost, Two-dimensional geometric variational problems, Wiley, Chichester, 1991. MR 92h:58045 Zbl 0729.49001

[Lamm 2010] T. Lamm, "Energy identity for approximations of harmonic maps from surfaces", Trans. Amer. Math. Soc. 362:8 (2010), 4077-4097. MR 2011d:58036 Zbl 1200.58016

[Li and Wang 2010a] Y. Li and Y. Wang, "Bubbling location for sequences of approximate $f$ harmonic maps from surfaces", Internat. J. Math. 21:4 (2010), 475-495. MR 2011g:58028 Zbl 1190.35067

[Li and Wang 2010b] Y. Li and Y. Wang, "A weak energy identity and the length of necks for a sequence of Sacks-Uhlenbeck $\alpha$-harmonic maps", Adv. Math. 225:3 (2010), 1134-1184. MR 2011j: 58022 Zbl 1203.58003

[Qing 1995] J. Qing, "On singularities of the heat flow for harmonic maps from surfaces into spheres", Comm. Anal. Geom. 3:1-2 (1995), 297-315. MR 97c:58154 Zbl 0868.58021

[Qing and Tian 1997] J. Qing and G. Tian, "Bubbling of the heat flows for harmonic maps from surfaces”, Comm. Pure Appl. Math. 50:4 (1997), 295-310. MR 98k:58070 Zbl 0879.58017

[Sacks and Uhlenbeck 1981] J. Sacks and K. Uhlenbeck, "The existence of minimal immersions of 2-spheres”, Ann. of Math. (2) 113:1 (1981), 1-24. MR 82f:58035 Zbl 0462.58014

[Sacks and Uhlenbeck 1982] J. Sacks and K. Uhlenbeck, "Minimal immersions of closed Riemann surfaces”, Trans. Amer. Math. Soc. 271:2 (1982), 639-652. MR 83i:58030 Zbl 0527.58008

[Simon 1983] L. Simon, Lectures on geometric measure theory, Proceedings of the Centre for Mathematical Analysis, Australian National University 3, Australian National University, Centre for Mathematical Analysis, Canberra, 1983. MR 87a:49001 Zbl 0546.49019

[Uhlenbeck 1989] K. Uhlenbeck, "Harmonic maps into Lie groups: classical solutions of the chiral model”, J. Differential Geom. 30:1 (1989), 1-50. MR 90g:58028 Zbl 0677.58020

[Valli 1988] G. Valli, "On the energy spectrum of harmonic 2-spheres in unitary groups", Topology 27:2 (1988), 129-136. MR 90f:58042 Zbl 0744.53027

Received September 29, 2013. Revised January 4, 2014.

\section{YUXIANG LI}

DEPARTMENT OF MATHEMATICAL SCIENCES

TSINGHUA UNIVERSITY

BEIJING, 100084

CHINA

yxli@math.tsinghua.edu.cn

YOUDE WANG

ACADEMY of MATHEMATICS AND Systems SCIENCES

CHINESE ACADEMY OF SCIENCES

BEIJING, 100080

CHINA

wyd@math.ac.cn 


\title{
PACIFIC JOURNAL OF MATHEMATICS
}

\author{
msp.org/pjm
}

Founded in 1951 by E. F. Beckenbach (1906-1982) and F. Wolf (1904-1989)

\section{EDITORS}

Don Blasius (Managing Editor)

Department of Mathematics

University of California

Los Angeles, CA 90095-1555

blasius@math.ucla.edu

\author{
Paul Balmer \\ Department of Mathematics \\ University of California \\ Los Angeles, CA 90095-1555 \\ balmer@math.ucla.edu \\ Robert Finn \\ Department of Mathematics \\ Stanford University \\ Stanford, CA 94305-2125 \\ finn@math.stanford.edu \\ Sorin Popa \\ Department of Mathematics \\ University of California \\ Los Angeles, CA 90095-1555 \\ popa@math.ucla.edu
}

\author{
Vyjayanthi Chari \\ Department of Mathematics \\ University of California \\ Riverside, CA 92521-0135 \\ chari@math.ucr.edu \\ Kefeng Liu \\ Department of Mathematics \\ University of California \\ Los Angeles, CA 90095-1555 \\ liu@math.ucla.edu \\ Jie Qing \\ Department of Mathematics \\ University of California \\ Santa Cruz, CA 95064 \\ qing@ cats.ucsc.edu
}

\section{PRODUCTION}

Silvio Levy, Scientific Editor, production@msp.org

\section{SUPPORTING INSTITUTIONS}

ACADEMIA SINICA, TAIPEI

CALIFORNIA INST. OF TECHNOLOGY

INST. DE MATEMÁTICA PURA E APLICADA

KEIO UNIVERSITY

MATH. SCIENCES RESEARCH INSTITUTE

NEW MEXICO STATE UNIV.

OREGON STATE UNIV.

\author{
STANFORD UNIVERSITY \\ UNIV. OF BRITISH COLUMBIA \\ UNIV. OF CALIFORNIA, BERKELEY \\ UNIV. OF CALIFORNIA, DAVIS \\ UNIV. OF CALIFORNIA, LOS ANGELES \\ UNIV. OF CALIFORNIA, RIVERSIDE \\ UNIV. OF CALIFORNIA, SAN DIEGO \\ UNIV. OF CALIF., SANTA BARBARA
}

\author{
Daryl Cooper \\ Department of Mathematics \\ University of California \\ Santa Barbara, CA 93106-3080 \\ cooper@math.ucsb.edu \\ Jiang-Hua Lu \\ Department of Mathematics \\ The University of Hong Kong \\ Pokfulam Rd., Hong Kong \\ jhlu@maths.hku.hk \\ Paul Yang \\ Department of Mathematics \\ Princeton University \\ Princeton NJ 08544-1000 \\ yang@math.princeton.edu
}

These supporting institutions contribute to the cost of publication of this Journal, but they are not owners or publishers and have no responsibility for its contents or policies.

See inside back cover or msp.org/pjm for submission instructions.

The subscription price for 2015 is US \$420/year for the electronic version, and \$570/year for print and electronic.

Subscriptions, requests for back issues and changes of subscribers address should be sent to Pacific Journal of Mathematics, P.O. Box 4163, Berkeley, CA 94704-0163, U.S.A. The Pacific Journal of Mathematics is indexed by Mathematical Reviews, Zentralblatt MATH, PASCAL CNRS Index, Referativnyi Zhurnal, Current Mathematical Publications and Web of Knowledge (Science Citation Index).

The Pacific Journal of Mathematics (ISSN 0030-8730) at the University of California, c/o Department of Mathematics, 798 Evans Hall \#3840, Berkeley, CA 94720-3840, is published twelve times a year. Periodical rate postage paid at Berkeley, CA 94704, and additional mailing offices. POSTMASTER: send address changes to Pacific Journal of Mathematics, P.O. Box 4163, Berkeley, CA 94704-0163.

PJM peer review and production are managed by EditFLOW ${ }^{\circledR}$ from Mathematical Sciences Publishers.

\section{PUBLISHED BY}

\section{mathematical sciences publishers \\ nonprofit scientific publishing}

http://msp.org/

(C) 2015 Mathematical Sciences Publishers 


\section{PACIFIC JOURNAL OF MATHEMATICS}

Volume $274 \quad$ No. $1 \quad$ March 2015

Unimodal sequences and "strange" functions: a family of quantum modular forms

KATHRIN BRINGMANN, AMANDA FOLSOM and ROBERT C.

RHOADES

Congruence primes for Ikeda lifts and the Ikeda ideal

JIM BROWN and RODNEY KEATON

Constant mean curvature, flux conservation, and symmetry

NiCK EDELEN and BRUCE SOLOMON

The cylindrical contact homology of universally tight sutured contact solid tori

ROMAN GOLOVKO

Uniform boundedness of $S$-units in arithmetic dynamics

Holly Krieger, Aaron LeVin, Zachary Scherr, ThOMAS

TUCKER, Yu YASUFuKu and MiCHAEL E. ZiEVE

A counterexample to the energy identity for sequences of $\alpha$-harmonic 107 maps

YUXIANG LI and YOUDE WANG

Theory of newforms of half-integral weight

Murugesan MANiCKAM, JABAN MEHER and BALAKRISHNAN RAMAKRISHNAN

Algebraic families of hyperelliptic curves violating the Hasse principle

NGUYEN NGOC DONG QUAN

$F$-zips with additional structure

RichaRd PINK, TORSTEN WEDHORN and PAUL ZiEgler

Mean values of $L$-functions over function fields 